\title{
LEGENDRE TRANSFORMATIONS OF CURVES
}

\section{SHLOMO STERNBERG}

In a recent paper of Wintner [1], an extension is made of a classical theorem on the Legendre transformation of a convex function (for details of the proof, see $[3]$, and for related results, cf. $[4 ; 5]$ ). His assumptions are that the function be strictly convex and of class $C^{1}$. Here we shall prove a more general result which eliminates both of these restrictions and shows that, in a sense, they are dual to one another. As two applications we mention a result of Kamke [2] on the Clairaut differential equation and a theorem on parallel curves (cf. [3, pp. 481-482]).

Let $y(x)$ be a convex bounded function of $x$ on the interval $(a, b)$. Then $y$ has monotone nondecreasing right- and left-hand derivates $y_{-}^{\prime}(x) \leqq y_{+}^{\prime}(x)$ in $(a, b)$. There will, in general, be two exceptional sets to consider in reference to these derivates, the set $j$ consisting of points $x$ where $y_{-}^{\prime}(x) \neq y_{+}^{\prime}(x)$ and the set $k$ of the closures of the $x$-intervals where $y_{+}^{\prime}(x)$ (or $y_{-}^{\prime}(x)$ ) is constant. $j$ is an at most denumerable set of points while $k$ is an at most denumerable set of closed intervals. Let $K$ be the set of closures of intervals of $y^{\prime}$-values satisfying $y_{-}^{\prime}(x)<y^{\prime}<y_{+}^{\prime}(x)$ for some $x$ on $j$, and $J$ the set of $y^{\prime}$-values for which $y^{\prime}=y^{\prime}(x)$ for some $x$ on $k$. Furthermore, let $A=y_{+}^{\prime}(a)$, $B=y_{-}^{\prime}(b)$.

We now consider the correspondence $X=y^{\prime}(x)$. This obviously assigns to every $x$ not in $j$ a unique $X$ not in $K$ and to every $X$ not in $J$ a unique $x$ not in $k$. Thus $X=y^{\prime}(x)$ is a unique (monotone and continuous) correspondence of $(a, b)-(j+k)$ onto $(A, B)-(J+K)$. In order to extend the domain of definition of this correspondence, put $\bar{x}=\bar{x}(X)=1$.u.b. $T(X)$, where $T(X)$ is the set of those $x$-values at which $y_{-}^{\prime}(x) \leqq X . \bar{x}(X)$ is in $T(X)$ and $\bar{x}(X)$ is continuous from the right.

We now define $Y=Y(X)$ as

$$
Y=\bar{x}(X) \cdot X-y(\bar{x}(X)) .
$$

Letting ' after $Y$ denote differentiation with respect to $X$ we have the following

Theorem. $Y_{+}^{\prime}$ and $Y_{-}^{\prime}$ exist everywhere and are nondecreasing with $Y_{-}^{\prime} \leqq Y_{+}^{\prime}$ and $Y_{+}^{\prime}(X)=\bar{x}(X)$. Furthermore, $Y_{-}^{\prime} \neq Y_{+}^{\prime}$ only on $J$ and $Y_{+}^{\prime}$ constant only on intervals of $K$.

Received by the editors April 19, 1954. 
In proving this theorem we make use of the following extension of the mean value theorem:

Lemma. If $f(t)$ has in $\left(t_{1}, t_{2}\right)$ a right-and left-hand derivate at every point and if $t^{\prime}<t^{\prime \prime}$ are points of $\left(t_{1}, t_{2}\right)$, then there exists a $t^{*}$ such that $t^{\prime}<t^{*}<t^{\prime \prime}$ and $\left(t^{\prime \prime}-t^{\prime}\right) f_{-}^{\prime}\left(t^{*}\right) \leqq f\left(t^{\prime \prime}\right)-f\left(t^{\prime}\right) \leqq\left(t^{\prime \prime}-t^{\prime}\right) f_{+}^{\prime}\left(t^{*}\right)$ or $\left(t^{\prime \prime}-t^{\prime}\right) f_{+}^{\prime}\left(t^{*}\right) \leqq f\left(t^{\prime \prime}\right)-f\left(t^{\prime}\right) \leqq\left(t^{\prime \prime}-t^{\prime}\right) f_{-}^{\prime}\left(t^{*}\right)$.

To see this set

$$
g(t)=f(t)-\left(t-t^{\prime}\right) \frac{f\left(t^{\prime \prime}\right)-f\left(t^{\prime}\right)}{t^{\prime \prime}-t^{\prime}} .
$$

$g(t)$ has right- and left-hand derivates in $\left(t^{\prime}, t^{\prime \prime}\right)$ and is certainly continuous on the closure of this interval. Furthermore $g\left(t^{\prime \prime}\right)=g\left(t^{\prime}\right)$ $=f\left(t^{\prime}\right)$ and therefore $g(t)$ assumes a maximum or a minimum in the interior of the interval, say at $t=t^{*}$. Then $g_{+}^{\prime}\left(t^{*}\right) \leqq 0, g_{-}^{\prime}(t) \geqq 0$ or $g_{-}^{\prime}\left(t^{*}\right)$ $\leqq 0, g_{+}^{\prime}\left(t^{*}\right) \geqq 0$ according as $t^{*}$ is a maximum or a minimum. This is the desired result.

We shall now show that $Y_{+}^{\prime}(X)$ exists everywhere and is equal to $\bar{x}(X)$. Let $X_{1}$ be greater than $X$ and $\Delta X=X_{1}-X, \Delta \bar{x}=\bar{x}\left(X_{1}\right)-\bar{x}(X)$, etc. Then

$$
\Delta Y=\bar{x}_{1} \Delta X+X \Delta \bar{x}-\Delta y(\bar{x}) .
$$

By the previous lemma, there exists an $x^{*}$ such that $\bar{x}<x^{*}<\bar{x}_{1}$ (unless $\bar{x}_{1}=\bar{x}$ in which case $\left.\Delta Y=\bar{x} \Delta X\right)$ and

$$
(\Delta \bar{x}) y_{-}^{\prime}\left(x^{*}\right) \leqq \Delta y \leqq(\Delta \bar{x}) y_{+}^{\prime}\left(x^{*}\right),
$$

so that

$$
\Delta Y \leqq \bar{x}_{1} \Delta X+X(\Delta \bar{x})-(\Delta \bar{x}) y_{-}^{\prime}\left(x^{*}\right) .
$$

But $X \leqq y_{-}^{\prime}\left(x^{*}\right) \leqq X_{1}$, hence

$$
\Delta Y \leqq \bar{x}_{1} \Delta X .
$$

Similarly,

$$
\Delta Y=X_{1} \Delta \bar{x}+\bar{x} \Delta X-\Delta y(\bar{x})
$$

and, since $x^{*}$ is strictly in between $\bar{x}$ and $\bar{x}_{1}$ (unless $\bar{x}=\bar{x}_{1}$ in which case $\Delta Y=\bar{x} \Delta X), X \leqq y_{+}^{\prime}\left(x^{*}\right) \leqq X_{1}$. Thus

$$
Y \geqq \tilde{x} \Delta X \text {. }
$$

We therefore have, by (2) and (3),

$$
\bar{x} \leqq \Delta Y / \Delta X \leqq \bar{x}_{1} .
$$


Letting $X_{1}$ tend to $X$ we get, since $\bar{x}$ is continuous from the right, $Y_{+}^{\prime}(X)=\bar{x}(X)$.

Since $\bar{x}$ is a nondecreasing function of $X, Y_{+}^{\prime}$ is nondecreasing. Furthermore, $Y_{-}^{\prime}(X)=Y_{+}^{\prime}(X-0)=\bar{x}(X-0), \quad Y_{-}^{\prime}(X) \leqq Y_{+}^{\prime}(X)$, and $Y_{-}^{\prime}(X)$ is nondecreasing. The points where $Y_{-}^{\prime}(X) \neq Y_{+}^{\prime}(X)$ are those where $\bar{x}(X-0) \neq \bar{x}(X)$ or where $y_{-}^{\prime}(x)$ is constant on some interval to the left of $\bar{x}$, thus belonging to $J$. Similarly $Y_{+}^{\prime}=\bar{x}$ is constant only on intervals of $K$.

$Y$ is thus a convex function of $X$. We can, therefore, apply the preceding transformation (1) on $Y=Y(X)$. We thus put $x=Y^{\prime}(X)$ on $[A, B]-(J+K)$ and $\bar{X}=\bar{X}(x)=$ l.u.b. $t(x)$ where $t(x)$ is the set of $X$ such that $Y_{-}(X) \leqq x$.

Finally, let $y^{*}=y^{*}(x)$ be defined by

$$
\begin{aligned}
y^{*} & =\bar{X}(x) \cdot x-Y[\bar{X}(x)] \\
& =\bar{X}(x) \cdot x-\bar{x}[\bar{X}(x)] \bar{X}+y(\bar{x}[\bar{X}(x)]) .
\end{aligned}
$$

Now, by the definitions of the functions $\bar{x}(X)$ and $\bar{X}(x), \bar{x}\left[\bar{X}\left(x_{0}\right)\right]$ is the largest $x$ such that $y_{-}^{\prime}(x) \leqq y_{-}^{\prime}\left(x_{0}\right)$. For $x$ not in $k, \bar{x}[\bar{X}(x)]=x$, in which case

$$
y^{*}=\bar{X} \cdot x-x \cdot \bar{X}+y(x)=y .
$$

For $x$ in an interval of $k, y(x)$ is linear with the constant slope $\bar{X}$ and assumes the value $y(\bar{x}[\bar{X}(\bar{x})])$ at $x=\bar{x}$, and so the last part of (4) shows that $y^{*}=y$.

Hence $y^{*} \equiv y$ for all $x$ in $[a, b]$. Thus the transformation (1) treated above is involutory with

$$
Y=Y(X)=\bar{x} X-y(\bar{x}), \quad Y_{+}^{\prime}(X)=\bar{x}
$$

and

$$
y=y(x)=\bar{X} x-Y(\bar{X}), \quad y_{+}^{\prime}(x)=\bar{X} .
$$

If $y$ has a derivative everywhere, then $j$ is empty and therefore $K$ is empty. Thus if $y$ is differentiable everywhere, $Y$ is strictly convex and vice versa. Similarly, if $y$ is strictly convex, the $Y$ has a derivative everywhere and vice versa. Finally, if $y$ or $Y$ are strictly convex and have derivatives everywhere, or if both are strictly convex or both are everywhere differentiable, then both are strictly convex and everywhere differentiable.

As a first application, we mention the following theorem:

The (outside) parallel curves of a convex curve are of class $C^{1}$ (at least). In effect, rolling a circle outside the curve increases the support 
function which means adding a strictly convex term to the Legendre transform (for suitable choice of coordinates).

As a second application, we mention the result of Kamke [2] on the Clairaut differential equation, namely, the existence of a nonlinear solution of $y=y^{\prime} x+f\left(y^{\prime}\right)$ when $f$ is strictly convex. Here, the Legendre transform of $f$ provides a solution if it is differentiable, which it must be in virtue of the strict convexity of $f$.

\section{REFERENCES}

1. A. Wintner, On the infinitesimal geometry of curves, Amer. J. Math. vol. 75 (1953) p. 258.

2. E. Kamke, Über die Clairautsche Differentialgleichung, Math. Zeit. vol. 27 (1928) pp. 623-639.

3. P. Hartman and A. Wintner, On pieces of convex surfaces, Amer. J. Math. vol. 75 (1953) pp. 477-487.

4. - On continuous area preserving and Legendre transformations, Amer. J. Math. vol. 76 (1954) pp. 87-96.

5. W. Fenchel, On conjugate convex functions, Canadian Journal of Mathematics vol. 1 (1949) pp. 73-77.

The Johns Hopkins University 